\title{
MÁS ALLÁ DE LOS DESAFÍOS DEL DECENIO DE LA EDUCACIÓN PARA EL DESARROLLO SOSTENIBLE: UNA REFLEXIÓN NECESARIA
}

\author{
A. C. SOUSA ${ }^{1}$, F. X. UCEDA-MAZA ${ }^{2}$ \\ ${ }^{1,2}$ Instituto Federal de Educação, Ciência e Tecnologia do Rio Grande do Norte, ${ }^{2}$ Universitat de València \\ alexsandra.sousa@ifrn.edu.br \\ Submetido 10/07/2017 - Aceito 05/09/2017 \\ DOI: 10.15628/holos.2017.6107
}

\section{RESUMEN}

La temática central del artículo se vincula a las discusiones llevadas a cabo por el Decenio de la Educación para el Desarrollo Sostenible (2005-2014) y tiene el objetivo de presentar las contribuciones del Decenio como herramienta de convergencia internacional que impulsó y fortaleció la comprensión de la educación como herramienta esencial para el desarrollo Sostenible y contribuyó a una percepción holística del estado mundial con la finalidad de generar actitudes y responsabilidades en la dirección del desarrollo sostenible, un modelo de desarrollo que satisface las necesidades del presente sin comprometer las necesidades de las futuras generaciones. Para ello, se discute y problematiza sobre los procedimientos y conceptos que antecedieron el Decenio, sus finalidades y objetivos inspiradores, además de discutir sobre las configuraciones actuales y perspectivas futuras más allá del Decenio de la Educación para el Desarrollo Sostenible.

PALAVRAS-CHAVE: Educación para el Desarrollo Sostenible, Decenio, futuras generaciones, percepción holística.

\section{BEYOND THE CHALLENGES OF THE DECADE OF EDUCATION FOR SUSTAINABLE DEVELOPMENT: A NECESSARY REFLECTION}

The major theme of this paper is connected to the discussions developed by the Decade of Education for Sustainable Development (2005-2014) and aims to present the Decade's contributions as a tool of international convergence that promoted and strengthened the understanding of education as an essential tool to the sustainable development and contributed to an holistic perception of the world with the purpose of fostering attitudes and responsibilities towards the sustainable development, a model of development that fulfills the necessities of the present without compromising the needs of the future generations. For that reason, there is a discussion and a problematization about the proceedings and concepts that came before the Decade, its purposes and the goals that worked as an inspiration for it, as well as a discussion about its current configurations and future perspectives beyond the Decade of Education for Sustainable Development.

KEYWORDS: Education for Sustainable Development, Decade, future generations, holistic perceptions. 


\title{
1 INTRODUCCIÓN
}

\begin{abstract}
"La interdependencia planetaria y la mundialización son fenómenos esenciales de nuestra época. Actúan ya en el presente y marcarán con su impronta el siglo XXI. Hoy hacen ya necesaria una reflexión global - que trascienda ampliamente los ámbitos de la educación y la cultura - sobre las funciones y las estructuras de las organizaciones internacionales" (La educación encierra un tesoro, Delors et al., 1996, p.31).
\end{abstract}

El escenario planetario de crisis ambiental al cual estamos inmersos, en función de un modelo económico insostenible de extracción de los bienes y servicios de la naturaleza, exige una reflexión-acción en el sentido de lograr un cambio radical que se constituya en un gran desafío para el ser humano en el siglo XXI. Un desafío que posibilite, sobre todo, la construcción de visiones de mundo capaces de percibir, comprender y aprender una nueva forma de convivencia entre los seres humanos y que conduzca, inevitablemente, a la reflexión sobre un proceso de educación capaz de retomar la génesis de la historia y desvelar los factores ligados directamente a las cuestiones actuales de degradación social y ambiental vinculados a la falencia del ideario de neutralidad y apoliticidad de la ciencia (Vilches \& Gil Pérez, 2016).

En esta línea de pensamiento, la Educación para el Desarrollo Sostenible emerge como una nueva ciencia que articula lo ambiental y lo social, que comporta nuevas racionalidades en la composición de una pedagogía de la complejidad ambiental (Leff, 2006), capaz de llevar a la internacionalización de las cuestiones ambientales y al comprometimiento ético y político con nuevas posturas y sentidos comunes ante las urgencias que se presentan para la construcción de la sostenibilidad económica, ecológica, social y cultural. Además, esta nueva ciencia se configura como un instrumento de transformación de la actual situación y representa una oportunidad para mejorar los sistemas educativos con el objetivo de incrementar una educación que acompañe de manera participativa y crítica los complejos procesos de cambios en nuestra sociedad.

Por ello, este trabajo se insiere dentro de las discusiones desarrolladas en el marco de la declaración del Decenio de la Educación para el Desarrollo Sostenible (2005-2014), una poderosa herramienta de convergencia internacional, cuyos objetivos, fundamentos, retos y oportunidades conducen a una reflexión sobre la necesidad de una Educación para el Desarrollo Sostenible solidaria, que contribuya a una percepción acertada del estado mundial y que sea capaz de generar compromisos y actitudes responsables para alcanzar el desarrollo sostenible.

Asimismo, la temática central de este trabajo está vinculada a la comprensión de la educación como herramienta esencial para el desarrollo sostenible, y tiene el objetivo de discutir las contribuciones de la Educación para el Desarrollo Sostenible en el marco del Decenio de la Educación para el Desarrollo Sostenible, presentando y problematizando los estudios y reflexiones sobre las perspectivas actuales y futuras de la educación para la sostenibilidad más allá de la nube de desafíos del Decenio de la Educación para el Desarrollo Sostenible. 


\section{EL DECENIO DE LA EDUCACIÓN PARA EL DESARROLLO SOSTENIBLE (DEDS): BREVE ANÁLISIS DE LOS PROCEDIMIENTOS Y DE LOS PRINCIPALES CONCEPTOS}

Muchas de las prácticas sociales que orientaban nuestras vidas desde hace pocos años han sido cambiadas por un nuevo escenario; un contexto donde los acontecimientos de la realidad nos han obligado a reestructurar la memoria colectiva y sus provisionales certidumbres. También hemos sido "obligados" a mirar con otros ojos el entorno que nos rodea, a modificar nuestros esquemas de pensamiento, a revisar nuestras formas de explicar y las maneras de entender lo que ocurre para actuar con conocimiento de causa. Pasamos a ser conscientes que los problemas ambientales representan el reto más difícil al que se enfrenta la humanidad en la Era Planetaria, ${ }^{1}$ y por ello, las agendas políticas mundiales, mediante normativas y convenios específicos, pasan a tener como una de sus prioridades la concretización del desarrollo sostenible dentro de las políticas públicas actuales de educación y de medio ambiente.

Por ello, destacamos la iniciativa llevada a cabo por la Organización de las Naciones Unidas para la Educación - UNESCO, al impulsar el Decenio de la Educación para el Desarrollo Sostenible (DEDS de ahora en adelante) (2005-2014) ${ }^{2}$, lo cual se configura como un instrumento de convergencia internacional muy relevante, que capacita a los ciudadanos/as a hacer frente a los retos del tiempo presente y futuro. Este instrumento, mediante una serie de objetivos, pretende conducir a la reflexión sobre la necesidad de una educación solidaria, que contribuya a una percepción acertada del estado mundial; que sea capaz de generar compromisos y actitudes responsables, que prepare a los ciudadanos/as para una toma de decisiones dirigidas al logro de un desarrollo culturalmente plural, socialmente justo y ecológicamente sostenible (Gutiérrez, et al., 2006).

A partir del momento en que el desarrollo sostenible pasa a ser apoyado por la Asamblea General de las Naciones Unidas, en 1987, el concepto de Educación para el Desarrollo Sostenible ${ }^{3}$ (EDS de ahora en adelante) también pasa a ser explorado. En la cumbre del Río, en 1992, se reunieron representantes de gobiernos, organizaciones internacionales, ONGs y la sociedad civil para discutir los desafíos del próximo siglo y adoptar un plan global de acción para enfrentarlos. Este plan de acción, conocido como Agenda 21, presentó una serie de principios para auxiliar gobiernos y otras instituciones en la implementación de políticas y programas para el desarrollo sostenible. Es en este momento histórico que la Comisión para el Desarrollo Sostenible confía a la UNESCO la responsabilidad de coordinar y hacer posible las metas y los retos propuestos en el

\footnotetext{
${ }^{1}$ Véase el Capítulo I “La Era Planetaria” de: Morin, E. \& Kern, A. B. (1993). Tierra-Patria. Barcelona: Kairós (pp. 15-43).

2 En diciembre de 2002, la Asamblea General de las Naciones Unidas proclama el Decenio de las Naciones Unidas de la Educación para el Desarrollo Sostenible (DEDS- 2005-2014), y confía a la UNESCO la elaboración de un Plan que enfatice el papel de la educación como herramienta indispensable para promover el desarrollo sostenible. Véase: <http://unesdoc.unesco.org/images/0014/001416/141629s.pdf>.

3 A lo largo de todo el trabajo utilizaremos el acrónimo "EDS" para referirnos al término "Educación para el Desarrollo Sostenible".
} 
capítulo 36 de la Agenda $21^{4}$, que centra su atención a la educación como herramienta esencial al desarrollo sostenible.

Es en el Capítulo 36 de la Agenda 21 donde están identificadas las tres grandes premisas de la EDS. La primera, consiste en reorientar la educación existente en todos los niveles en dirección al desarrollo sostenible, de manera que se pueda repensar y revisar la educación desde la guardería hasta la universidad para incluir principios, habilidades, perspectivas y valores relacionados con la sostenibilidad en cada una de las tres esferas - social, ambiental y económica - importantes para las sociedades actuales y futuras. Las dos últimas premisas, expresan la importancia de desarrollar una atención al público con la finalidad de promover la conciencia sostenible y fomentar la capacitación. Consideran que para que haya un avance en la construcción de sociedades más sostenibles es necesario una población que sea consciente de los objetivos de las sociedades sostenibles y que tengan conocimientos y habilidades para contribuir con estos.

El Decenio, además de recordar las premisas establecidas en el Capítulo 36, refleja su preocupación por una educación, en la que el desarrollo sostenible forme parte integral de sus planes de estudio, promoviendo la reflexión crítica, el aprendizaje de valores y la capacidad de tomar decisiones mediante la participación. Para ello, exige una estrecha cooperación entre los diversos sistemas e instituciones territoriales en los niveles local, nacional, regional e internacional, en los que la creación de redes y de foros de comunicación ha de constituir el elemento determinante de los avances en la misma dirección.

Se articula como un instrumento de coordinación programática, con la finalidad de establecer vínculos estrechos entre programas en curso, tales como el Decenio de las Naciones Unidas de la Alfabetización, el Programa de Educación para Todos, y los Objetivos de Desarrollo del Milenio. Propone a los órganos gubernamentales y no gubernamentales la formulación de políticas, marcos, consultas y aportaciones de los ciudadanos/as, organización de campañas públicas, integración y puesta en práctica de la sostenibilidad en las instituciones, en la administración pública y, principalmente, en los sistemas educativos. De acuerdo con Gutiérrez (op.cit., p.23):

El Decenio de las Naciones Unidas para la Educación con miras al Desarrollo Sostenible, pretende promover la educación como fundamento de una sociedad más viable para la humanidad, e integrar el desarrollo sostenible en el sistema de enseñanza a todos los niveles. El Decenio intensificará igualmente la cooperación internacional en favor de la elaboración y de la puesta en común de prácticas, políticas y programas innovadores de educación para el desarrollo sostenible.

La intencionalidad del DEDS es contribuir para un futuro que concilie las necesidades humanas con el uso sostenible de los recursos naturales, superando así, los efectos devastadores, desde la destrucción del ambiente hasta la agravación de la pobreza. En este sentido, el DEDS tiene una dimensión muy idealista, cuyo horizonte de interés sólo puede ser asumido como un cuadro orientador de valores, que produzca la búsqueda por vías pragmáticas para alterar el

\footnotetext{
${ }^{4}$ Véase Capítulo 36 de la Agenda 21: <http://portal.mec.gov.br/seb/arquivos/pdf/c36a21.pdf>.
} 
sentido de los procesos que están conduciendo a la ruptura generalizada en el medio ambiente y en la sociedad.

El Decenio de la Educación para el Desarrollo Sostenible posee en su esencia una idea simple con implicaciones complejas: después de vivir siglos sin preocuparnos con el agotamiento de los recursos naturales del planeta, ahora, tenemos que aprender a vivir de forma sostenible (Aguilar, 2017). La mayor parte de este desafío, consiste en estimular cambios de actitudes y comportamientos en la sociedad mundial, ya que nuestras capacidades intelectuales, morales y culturales imponen responsabilidades para todos los seres vivos que coexisten en el planeta. La DEDS, esencialmente, es un instrumento de movilización, difusión e información y una red de responsabilidades donde los gobiernos, organizaciones internacionales, sociedad civil, sector privado y comunidades locales alrededor del mundo, pueden demonstrar su compromiso práctico de aprender a vivir la sostenibilidad.

\subsection{Finalidades y objetivos inspiradores del Decenio de la Educación para el Desarrollo Sostenible}

La Educación para el Desarrollo Sostenible es un "concepto dinámico que comprende una nueva visión de educación que busca empoderar a las personas de todas las edades para asumir la responsabilidad de crear y desfrutar de un futuro sostenible" (Unesco, 2002) ${ }^{5}$, con la finalidad de otorgar poderes a los ciudadanos/as para luchar por cambios sociales y ambientales positivos, implicándoles en una acción participativa con los demás sujetos involucrados. Además, educar para la sostenibilidad, implica integrar conceptos y herramientas analíticas de una variedad de disciplinas para auxiliar a las personas a comprender mejor el mundo en que viven. Perseguir el desarrollo sostenible a través de la educación exige que los educadores/as y educandos reflexionen críticamente en sus propias comunidades, identifiquen elementos inviables en sus vidas, y exploren tensiones entre valores y objetivos.

En este sentido, las finalidades que inspiraron el DEDS están basadas en prescripciones que buscan proporcionar a las personas el derecho a una vida saludable y productiva en armonía con la naturaleza. La primera finalidad, está orientada a la concienciación de los países al explotar sus propios recursos sin causar daños ambientales más allá de sus fronteras. En el caso de que hagan daño a su medio, debido a las actividades bajo su control, deben tener leyes que concretamente ofrezcan compensaciones por los prejuicios que muchas veces son irremediables.

La importancia de tal finalidad reside en el refuerzo de que sólo se puede lograr el desarrollo sostenible, mediante la protección ambiental como parte integral del proceso de desarrollo, y no como un elemento aislado. La segunda finalidad, tiene como prioridad la erradicación de la pobreza y la reducción de las disparidades entre los estándares de vida en diferentes partes del mundo para lograr el desarrollo sostenible y satisfacer las necesidades de la mayoría de las personas. De acuerdo con ésta, las naciones deben cooperar para conservar, proteger y restaurar la salud e integridad del ecosistema terrestre, siendo que los países

\footnotetext{
${ }^{5}$ Unesco (2002). Education for Sustainability-from Rio to Johannesburg: Lessons Learnt from a Decade of Commitment. Véase: <http://unesdoc.unesco.org/images/0012/001271/127100e.pdf>.
} 
desarrollados deben reconocer y hacer valer sus responsabilidades en la búsqueda internacional del desarrollo sostenible en vista de las presiones que sus sociedades imponen al medio ambiente global y de las tecnologías y recursos financieros que dominan.

La tercera y última finalidad del DEDS, consiste en incentivar la participación de todos los ciudadanos/as interesados en las cuestiones ambientales. Para ello, todas las naciones deberán facilitar y fomentar la conciencia y participación pública poniendo la información ambiental a disposición de todos/as, a través de leyes ambientales efectivas, como las de protección a las víctimas de la contaminación y otros daños de carácter ambiental.

El enfoque que se promueve en los debates y en los documentos que fundamentaron el DEDS, están centrados en la construcción de un mundo en donde todos/as las personas tengan las mismas posibilidades de beneficiarse de las oportunidades que ofrecen la educación para el aprendizaje de valores, de comportamientos y de estilos de vida requeridos por un futuro sostenible. Para cumplir con estas aspiraciones, el DEDS traza los siguientes objetivos:

- Promover un intenso desarrollo del papel central de la educación y del aprendizaje en el objetivo común del desarrollo sostenible;

- Favorecer las relaciones y las redes, el intercambio y la interacción entre los responsables del EDS;

- Crear espacios y oportunidades para redefinir y para promover una visión de transición hacia el desarrollo sostenible mediante todas las modalidades posibles de aprendizaje y de desarrollo;

- Fomentar una mayor calidad de la enseñanza y del aprendizaje en la educación al servicio del desarrollo sostenible;

- Impulsar estrategias a todos los niveles para desarrollar capacidades en EDS.

En conformidad con los objetivos del Decenio, la Organización de los Estados Iberoamericanos-OEI (Gutiérrez, op.cit. p.25) también centra su atención en la lucha por una educación solidaria, que contribuya a una correcta percepción del estado del mundo y que genere actitudes y comportamientos responsables. Para ello, establece objetivos que consisten en:

- Contemplar los problemas ambientales y del desarrollo en su globalidad, teniendo en cuenta las repercusiones a corto, medio y largo plazo, tanto para una determinada colectividad como para el conjunto de la humanidad y de nuestro planeta;

- Comprender que no es sostenible un éxito que exija el fracaso de otros;

- Transformar la interdependencia planetaria y la mundialización en un proyecto plural, democrático y solidario;

- Reivindicar e impulsar desarrollos tecnocientíficos favorecedores de la sostenibilidad, con control social y con la aplicación sistemática del principio de precaución; 
- Implantar acciones sociopolíticas en defensa de la solidaridad y de la protección del medio, a escala local y planetaria, que contribuyan a poner fin a los desequilibrios insostenibles y a los conflictos asociados, con una decidida defensa de la ampliación y de la generalización de los derechos humanos al conjunto de la población mundial, sin discriminaciones de ningún tipo (étnicas, de género, etc.);

- Superar la defensa de los intereses y de los valores particulares, y promover la comprensión de que la solidaridad y la protección global de la diversidad biológica y cultural constituyen requisitos necesarios para una auténtica solución de los problemas.

La Unión Internacional para la Conservación de la Naturaleza (UICN), ${ }^{6}$ también ha desarrollado iniciativas en este sentido, orientadas al fomento de los objetivos del Decenio, las cuales han sido desarrolladas para proporcionar un conocimiento, por parte del público, de los principios en los que se asienta la sostenibilidad. Además, La UICN afirma que la EDS juega un papel que va más allá de la propia discusión sobre la sostenibilidad, para pasar de la evolución del concepto a una visión en la que lo relevante es cómo se aplica en la práctica a los distintos contextos culturales y locales. Para ello, considera necesario la implicación de todos los sectores sociales, incluidas las empresas, el sector agrícola, el turismo, los gestores de los recursos naturales, los gobiernos locales, los medios de comunicación en el desarrollo y en la implementación de los programas.

\section{LAS CONFIGURACIONES ACTUALES Y PERSPECTIVAS FUTURAS DEL DECENIO DE LA EDUCACIÓN PARA EL DESARROLLO SOSTENIBLE}

El panorama al que se afrontó el Decenio en la segunda etapa de su desarrollo (20102015), no se ha revelado nada esperanzador. La crisis financiera y económica mundial a que todos/as todavía estamos envueltos, nos hace ver la notoriedad de las deficiencias de los paradigmas económicos dominantes, obligándonos a repensar nuestros estilos de vida, los modelos de desarrollo y los valores personales que hasta poco adoptábamos. La forma repentina y violenta en que emergió el contexto de crisis vino a romper con la falsa estabilidad y la idea de continuidad del "progreso". De acuerdo con Martínez Bonafé (2011, p.1),

(...) el dramático contexto de capitalismo feroz que, en su fase actual, bajo el paraguas discursivo de "la crisis," acentúa y desenmascara, más todavía, la voracidad sin límites de unos pocos y el creciente empobrecimiento económico y la idiotización cultural de muchos. Un pequeño número de instituciones financieras y corporaciones globales condicionan, a través de eso que llaman "los mercados," las posibilidades de supervivencia de millones de seres humanos, hipotecando, despilfarrando o poniendo en peligro, además, los alimentos, el agua, los combustibles, el territorio, que necesitarán para su supervivencia en el planeta las generaciones futuras

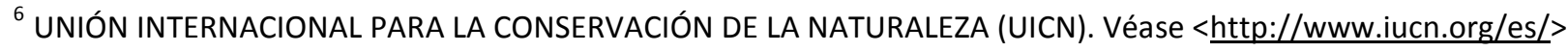


¿Qué respuestas esperamos de la EDS y del DEDS en relación a las realidades cambiantes que la rodean? ¿Cómo éstas contribuirán a un futuro mejor? Con la pretensión de responder a estas preguntas, se elaboró la Estrategia de la Unesco para la Segunda Mitad del Decenio de las Naciones Unidas de la EDS (Unesco, 2010) ${ }^{7}$, que se basa en las conclusiones y enseñanzas extraídas de la primera fase del proceso de seguimiento y evaluación del Decenio, mencionadas en el informe de 2009 titulado "El aprendizaje para un mundo sostenible: análisis de los contextos y estructuras de la EDS 2009: principales conclusiones y camino a seguir" (Unesco, 2009) ${ }^{8}$, fruto del compromiso de la Unesco en garantizar que los propósitos del Decenio en lograr el aprendizaje para un mundo sostenible se cumpliesen debidamente. Para eso, se comprometió a elaborar una serie de informes de seguimiento y evaluación, los cuales deberían ser publicados en los años 2009, 2011 y 2015.

El informe de seguimiento y evaluación del Decenio de 2009 analiza los primeros cinco años del Decenio (2005-2010), apuntando los avances y los obstáculos que han surgido, en la elaboración de disposiciones, estrategias, mecanismos y contextos que respaldan el fomento y la aplicación de la EDS. En él, fueron presentadas las conclusiones de este primer periodo e indicadas las líneas de actuación para la segunda mitad del Decenio. Las principales conclusiones mencionadas en el informe consistieron en explicar los significados de la EDS, teniendo en cuenta sus distintas interpretaciones de acuerdo con las diferencias regionales, nacionales y locales y el hecho de que, a pesar de que se deben estimular ampliamente las distintas interpretaciones de la EDS y del Desarrollo Sostenible (Hernández, 2016), la importancia de las interrelaciones entre las dimensiones de orden económico, medioambiental, social y cultural del desarrollo debe ser el elemento común presente en todas las actividades de educación y aprendizaje para el desarrollo sostenible.

Además, el informe de seguimiento referente a la segunda mitad del Decenio llama la atención para las contribuciones de las Naciones Unidas al Decenio: EI DEDS constituye una iniciativa de las Naciones Unidas que se caracteriza por el compromiso de varios organismos del sistema para la realización de la EDS. Los actos de inauguración del Decenio en los planos internacional, regional, nacional y local, los talleres y reuniones relacionados con la EDS y el gran número de publicaciones sobre ese tema son sólo algunas de las muchas actividades e iniciativas emprendidas por los organismos de las Naciones Unidas en el marco de implementación del Decenio.

Sin embargo, queda mucho por hacer para que se haga realidad una respuesta concertada de las Naciones Unidas enmarcada en la Iniciativa "Unidos en la acción". En este sentido, el refuerzo de los recursos financieros y humanos podría contribuir a mejorar la ejecución de las actividades del Decenio, ya que para implementar la EDS en un país resulta de vital importancia la existencia de un órgano nacional de coordinación de la EDS, donde el compromiso forme parte de las políticas educativas nacionales y los planes de estudios desde la enseñanza primaria hasta la

\footnotetext{
${ }^{7}$ Unesco (2010). Principales ejes de acción para la segunda mitad del Decenio de las Naciones Unidas de la Educación para el Desarrollo Sostenible (2010-2015). Véase: <http://unesdoc.unesco.org/images/0021/002154/215466s.pdf>.

${ }^{8}$ Unesco (2009). Análisis de los contextos y estructuras de la Educación para el Desarrollo Sostenible 2009: Principales Conclusiones y Caminos a Seguir. Recuperado de: <http://unesdoc.unesco.org/images/0018/001877/187757s.pdf >.
} 
educación superior. Incluir la sostenibilidad en los programas de estudios actuales, adoptar nuevos enfoques de enseñanza tales como la enseñanza y el aprendizaje interdisciplinarios y rediseñar los planes de estudios, son algunas de las modalidades que los países deben cumplir para integrar la EDS en los contextos educativos formales, además de investir en la consolidación de los proyectos existentes en los niveles de educación no formal e informal (Huerta, Colás \&Valentí, 2016).

Además de exponer las principales conclusiones, el informe también define los 10 (diez) ámbitos de acción para la segunda mitad del Decenio (2010-2014), con los siguientes objetivos: a) promover la sensibilización con la EDS; b) reorientar los programas de estudios, la enseñanza y el aprendizaje hacia el desarrollo sostenible; c) desarrollar las capacidades profesionales en materia de EDS; d) respaldar la investigación, el seguimiento y la evaluación relacionados con la EDS; e) establecer una sinergia entre la EDS y otras temáticas; f) reforzar la cooperación internacional y regional y las redes interregionales e intrarregionales para el cumplimiento del programa de la EDS; g) reforzar las redes nacionales y la coordinación entre diversos ministerios e instancias del gobierno para la promoción de la EDS en el plano nacional; y por último h) aumentar la financiación de la EDS, mediante la recaudación de fondos para las actividades y proyectos de educación para la sostenibilidad.

¿Y ahora qué? ¡Una nube de compromisos! $!^{9}$ Este ha sido el título del 850 Boletín de la OEI a respecto del Decenio. En él, se reconoce el fracaso de las expectativas esperadas para los últimos años del Decenio (2013 y 2014) y la ausencia de avances significativos hacia la sostenibilidad:

La falta de compromisos vinculantes para hacer frente a la actual situación de emergencia planetaria fue particularmente decepcionante en la Cumbre Rio+20 sobre Desarrollo Sostenible: el documento «El futuro que queremos» aprobado a su término solo contiene buenas intenciones y vagas promesas. Así lo denunció, entre otros, el Grupo Principal de Comunidades de Ciencia y Tecnología (una de las nueve comunidades que tuvieron acceso oficial al proceso de negociación), lamentando que el documento final no se refiriera al concepto de "límites planetarios", ni se hubiera aprovechado la oportunidad para expresar la "profunda alarma» de la comunidad científica sobre el estado de los recursos del planeta, el aumento continuo de las emisiones de gases de efecto invernadero, la inseguridad alimentaria, etc. [además], se lamentó, sobre todo, que no se hubieran sentado las bases para una urgente transición hacia una economía baja en carbono y solidaria, que evite los desastres ecológicos y sociales y haga posible un futuro sostenible. (Boletín no 85 de la OEI, 2013, p.1)

El Boletín denuncia la falta de la voluntad e implicación política, en la representación del conjunto de la sociedad, donde se verifica una indiferencia con los retos planteados por el Año Internacional de la Energía Sostenible para todos/as y con la Cumbre de la Tierra Río+20, dejando que la actual crisis financiera los oscurezca y los destine al olvido:

\footnotetext{
${ }^{9}$ Clound of Commitments: Action and accountability at the Rio+20. Earth Summit and Beyond. Recuperado de: <http://www.oei.es/decada/boletin085.php $>$.
} 
Parece no haberse comprendido todavía que la actual crisis sólo puede resolverse en la medida en que demos paso a un nuevo modelo económico que propicie un desarrollo humano realmente sostenible. Un desarrollo que ponga en marcha una profunda transición energética, hoy técnicamente posible, para hacer accesibles a todos recursos energéticos limpios y renovables y reducir drásticamente las emisiones de efecto invernadero. Un desarrollo que posibilite la gestión racional y sostenible de los recursos básicos y la estabilización de la población por debajo de la capacidad de carga del planeta; que garantice la seguridad alimentaria y la erradicación de la pobreza; que disminuya los inaceptables desequilibrios actuales, etc. Es preciso comprender y hacer comprender que la construcción de un futuro sostenible no es algo que pueda posponerse para ocuparse ahora de «lo urgente», es decir, de la crisis económica. Es, por el contrario, la única vía para superar esta crisis, que no es solo económica sino socioambiental y que se agrava aceleradamente. (op. cit., p.1)

Los últimos años del Decenio traen consigo un gran desafío para el campo de la EDS: la necesidad que no olvidemos los compromisos firmados al principio, lo cual han sido fruto de un proceso histórico de luchas en favor de la Educación Ambiental para el Desarrollo Sostenible. Tenemos a nuestra disposición herramientas como "La nube de compromisos," una página web creada con la finalidad de hacer presión positiva en relación a los problemas que tenemos que enfrentar en estos últimos años del DEDS.

Además, como respuesta a los resultados del Río+20, el Secretario General de las Naciones Unidas, Ban Ki-moon, lanzó en agosto de 2012, una red de centros de investigación, donde participan universidades e instituciones técnicas, con la finalidad de encontrar soluciones a los problemas que ejercen más presión a nivel socioeconómico sobre el medio ambiente, la Red de Soluciones para el Desarrollo Sustentable, ${ }^{10}$ que manifiesta su preocupación en relación a las causas que la actual situación socioeconómica puede representar en el camino hacia el Desarrollo Sostenible:

The scale of the global sustainable development challenge is unprecedented. The fight against extreme poverty has made great progress under the Millennium Development Goals (MDGs), but more than 1 billion people continue to live in extreme poverty. Inequality and social exclusion are widening within most countries. As the world population is estimated to rise to 9 billion by 2050 and global GDP to more than US\$200 trillion, the world urgently needs to address the sustainable development challenges of ending poverty, increasing social inclusion, and sustaining the planet. (Sustainable Development Solutions Network, 2012, p.1).

Por ello, la Red de Soluciones para el Desarrollo Sostenible manifiesta como una de sus prioridades el compromiso de:

Over the coming twelve months the Solutions Network will be built up to mobilize scientific and technical expertise from academia, civil society, and the private

10 Sustainable Development Solutions Network. A Global initiative for the United Nations. Recuperado de: <http://www.unsdsn.org $>$. 
sector in support of sustainable-development problem solving at local, national, and global scales. This Solutions Network will accelerate joint learning and help to overcome the compartmentalization of technical and policy work by promoting integrated approaches to the interconnected economic, social, and environmental challenges confronting the world. The SDSN will work closely with United Nations agencies, multilateral financing institutions, as well as other international organizations. [...] The Network will convene global expert Thematic Groups on key sustainable development challenges that will identify common solutions and highlight best practices. It will also provide technical support to the High-level Panel of Eminent Persons on the Post-2015 Development Agenda. [...] Over time the SDSN will launch projects to pilot or roll-out solutions to sustainable development challenges and assist countries in developing sustainable long-term development pathways. (op.cit. p. 1)

La creación de redes como éstas, gubernamentales o no, ciertamente aportan contribuciones que nos ayudarán a alcanzar los objetivos propuestos por el Decenio y contribuyen con el propósito de profundizar los estudios e reflexiones sobre las perspectivas actuales en el contexto de la EDS, propiciando los "anteojos" críticos necesarios al análisis de programas e proyectos necesarios a la construcción de un marco teórico más amplio sobre los principios pedagógicos de la EDS (García \& Muñoz, 2013; Caurín et al., 2012; Tilbury, 2006, 2007, 2009 y 2012; Hopkins, 2012; Huckle, 2008, 2012; Vilches \& Gil Pérez, 2015).

En este sentido, pensar más allá del Decenio de la Educación para el Desarrollo Sostenible nos "obliga," irremediablemente, al ejercicio de resignificación de la mirada delante del desafío de implementar a nivel mundial una nueva tendencia educativa, que requiere un liderazgo responsable y una experiencia tanto en relación a los cambios en el sistema educativo, como en relación a la comprensión crítica del desarrollo sostenible. Un cambio que requiere, sobre todo, que las instituciones educativas en todos sus niveles reorienten la educación vigente hacia la inclusión de la EDS en los planes curriculares.

Sin embargo, es cierto que la dificultad en hacer posible reformas educativas eficaces debido a la "imposibilidad lógica" o el "duplo bloqueo" (Morin, 2004), "penetra" en el camino del Decenio como inmenso obstáculo, ya que tal "imposibilidad lógica" consiste en defender la idea de que no se puede reformar una institución sin una previa reforma de las mentes, tampoco se puede reformar las mentes, sin una previa reformas de las instituciones. Así, Edgar Morin nos indica que para superar este problema, es necesario empezar de alguna forma, hasta que la idea sea diseminada y, cuando se difunda, se convierta en una fuerza actuante.

\section{CONCLUSIONES}

Pensar sobre la Educación para el Desarrollo Sostenible en el contexto de la modernidad liquida (Bauman, 2005) significa pensar en sus correlaciones con el conocimiento científico; pensar en los vínculos que forman las características de las instituciones educativas y su organización curricular (propuestas curriculares); significa pensar cómo se organiza la sociedad actual y las implicaciones del sistema capitalista para el medio socioambiental. En este contexto político, 
científico y cultural de la problemática socioambiental, la EDS se configura como un área de una sólida historia en los debates científicos y epistemológicos.

A pesar de todas las dificultades, la Educación para el Desarrollo Sostenible ofrece elementos consistentes para mostrar que es una ciencia que se consolida cada vez más y presenta visiones alternativas y paradigmas consecuentes para la construcción de una sociedad sostenible (Leff, 2002; 2006; Vilches \& Gil Pérez, 2016). Además, es un área que propone el análisis de su trayectoria y recepción de investigadores (as) para desvelar el proceso de constitución y consolidación de conceptos y concepciones como sostenibilidad y la propia comprensión del Decenio de la Educación para el Desarrollo Sostenible.

El Decenio dialoga con los diversos conocimientos contemporáneos producidos por la ciencia en dialogo con los conocimientos populares y étnicos construidos en el cotidiano a lo largo de la historia. Sin embargo, el decenio tiene un fuerte componente utópico, que nos lleva a cuestionar, si en estas realidades de tantas desilusiones con el sistema y sus instituciones, la ciencia para la sostenibilidad tendrá condiciones de reunir nuevos investigadores (as) dispuestos a innovar y afrontar el actual modelo de sociedad. ¿Cómo actuar delante de la "toda poderosa" ciencia direccionada al desarrollo bélico y económico, que quiere ser la única y hegemónica detenedora de los principios y métodos "correctos" racionales y adecuados?

En este sentido, es necesario tener la seguridad de que optar por la "ciencia sostenible" implica romper con un estilo de vida que puede costar muy caro a los sujetos e instituciones que de ella adherir. Implica transitar por un área científica y política con altos riesgos e incertidumbres. La ciencia en la perspectiva de la sostenibilidad es una ciencia que experimenta, que busca, que duda de los resultados y de su aplicación, pero que está convencida de su importancia, pertinencia y compromiso político.

Pensamos la ciencia no en la perspectiva de la no neutralidad, lo que implica asumir la comprensión de que el investigador también aprende al investigar y que la trayectoria y selección del objeto de estudio pueden facilitar elementos sólidos para mostrar que, a pesar de las dificultades, otra modalidad de ciencia continuará consolidándose en el camino de la EDS. Así, investigar sobre la EDS en el contexto del Decenio significa caminar por un campo que surge como un elemento preponderante entre un futuro sostenible o un futuro caótico (Leff, 2001; Vilches \& Gil Pérez, 2005).

La investigación en el campo de la EDS contribuye para fomentar la participación de toda la comunidad educativa y grupos sociales en la reflexión sobre las configuraciones de la educación actual y sus demandas, de modo que podamos tomar decisiones conscientes en la dirección de un mundo más sostenible. Contribuye para potenciar un coyuntura necesaria que provoque la creación de sentidos críticos, que conduzca a la comprensión de la dimensión ambiental como un proceso que incluye un conjunto de actores del universo educativo, con el propósito de potencializar el compromiso por parte de todos ellos de forma integral. Implica pensar la realidad bajo el paradigma de la complejidad y definirla como una nueva racionalidad y un espacio donde se articulan naturaleza, sociedad, valores y cultura.

La Educación para el Desarrollo Sostenible emerge solicitando una nueva ciencia que articule lo ambiental y lo social, que comporte nuevas racionalidades (la reflexiva y la ambiental), 
en la composición de una pedagogía de la complejidad ambiental (Leff, op.cit.), capaz de llevar a la internacionalización de las cuestiones ambientales y al comportamiento ético y político con nuevas posturas y sentido comunes delante de las urgencias que se presenten para la construcción de la sostenibilidad económica, ecológica, social y cultural; sostenibilidades que, con el proceso de globalización de los riegos (Beck, 2002), pasa a ser de carácter planetario.

De esta manera, ¿̇lo que hay de nuevo después del Decenio de la Educación para el Desarrollo Sostenible? ¿Qué hacer delante de la nube de compromisos hacia la implementación de la Educación para el Desarrollo Sostenible? Con estas cuestiones cerramos este artículo, con el deseo de cambios significativos que ayuden a lograr los muchos desafíos que tenemos por delante. Asimismo, este trabajo se configura como una herramienta de discusión del futuro de la Educación para el Desarrollo Sostenible, corroborando con el grande desafío del Programa de las Naciones Unidas para el Desarrollo (2015) al proclamar la Agenda 2030 con los Objetivos del Desarrollo Sostenible.

\section{REFERENCIAS}

Aguilar, E.C. (2017) Cambio climático, adaptación y seguridad humana: vínculos esenciales. Delos: Desarrollo Local Sostenible. 10(28): 21 p.

Bauman, Z. (2005). Modernidad líquida. Argentina: Fondo de Cultura Económica.

Beck, U. (2002). La sociedad del riesgo global. Madrid, Siglo XXI.

Caurín, C., (2012). ¿Es posible un cambio de actitudes hacia un modelo de desarrollo sostenible? Didáctica de las Ciencias Experimentales y Sociales, (26): 229-245. DOI: 10.7203/DCES.26.1926

García, A. \& Muñoz. J.M. (2013). Enfoques tradicionales y enfoques emergentes en la construcción del marco teórico de la educación ambiental para el desarrollo sostenible. Revista Española de Pedagogía. Vol. 71, no 255, pp.209-226.

Gutiérrez, J., Benayas, J. \& Calvo, S. (2006). Educación para el desarrollo sostenible: evaluación de retos y oportunidades del Década 2005-2014. Revista Iberoamericana de Educación. (40): 25-69.

Hernández. D.H (2016). Consideraciones teóricas acerca del concepto de desarrollo. Delos: Desarrollo Local Sostenible. 9(27): 26 p.

Hopkins, C. (2012). Reflections on 20+ Years of ESD. Journal of Education for Sustainable Development, v 6 n1, 21-35. ERIC Number: EJ964584.

Huckle, J. (2008). An Analysis of New Labour's Policy on Education for Sustainable Development with Particular Reference to Socially Critical Approaches. Environmental Education Research, v 14, n1, 65-75. 
Huerta, R.M.M; Colás, R.H. \& Valentí, C.M. (2016). Una propuesta evaluativa para actividades de educación ambiental para la sostenibilidad. Educación XXI. Revista de la Facultad de Educación. 19(1): 331-355.

Informe a la Unesco de la Comisión Internacional sobre la Educación para el Siglo XXI (1996). La Educación encierra un tesoro. Madrid: Ediciones UNESCO. Disponible en: $<$ www.unesco.org/education/pdf/DELORS S.PDF>.

Leff, E. (2001). Epistemologia ambiental. São Paulo: Cortez.

. (2002). Saber ambiental: sustentabilidad, racionalidad, complejidad y poder. 3aed, México: Siglo Veintiuno.

- (2006). Complejidad, racionalidad ambiental y diálogo de saberes. Barcelona: I Congreso internacional interdisciplinar de participación, animación e intervención socioeducativa. CNEA.

Martínez Bonafé, J. (2012). El problema del conocimiento en el triángulo entre capitalismo, crisis y educación. Revista Investigación en la Escuela, vol 76, p. 07-21.

Morin, E. y Kern, A.B. (1993). Tierra-Patria. Barcelona: Kairós.

Morin, E. (2004). A cabeça bem feita: repensar a reforma, reformar o pensamento. 9a ed. Rio de Janeiro: Bertrand Brasil.

Programa de las Naciones Unidas para el Desarrollo (PNUD). Objetivos del Desarrollo Sostenible (Agenda 2030). Disponible en: <http://www.undp.org/content/undp/es/home/sustainabledevelopment-goals.html>.

Unesco (2002). Education for Sustainability, From Rio to Johannesburg: Lessons Learnt from a Decade of Commitment. UNESCO, París.

- (2009). Análisis de los contextos y estructuras de la Educación para el Desarrollo Sostenible 2009: Principales Conclusiones. UNESCO, París.

. (2010). Principales ejes de acción para la segunda mitad del Década de las Naciones Unidas de la EDS (2010-2015). UNESCO, París.

Tilbury, D. (2006). Australia's Response to a UN Decade in Education for Sustainable Development. Australian Journal of Environmental Education, v 22, n1, 77-81. ERIC Number: EJ843349.

. (2007). Monitoring and Evaluation during the UN Decade of Education for Sustainable Development. Journal of Education for Sustainable Development, v1, n2, p. 239-254. ERIC Number: EJ911264.

. (2012). Tracking Our Progress: A Global Monitoring and Evaluation Framework for the UN DESD. Journal of Education for Sustainable Development, v3, n2, p189-193.

Vilches, A., Gil Pérez, D. \& Oliva, J.M. (2005). Década de la Educación para el Desarrollo Sostenible. Algunas ideas para elaborar una estrategia global. Revista Eureka sobre Enseñanza y Divulgación de las Ciencias, vol. 2, n 1, 91-100. 
Vilches, A. \& Gil Pérez, D. (2015). Ciencia de la Sostenibilidad: ¿Una nueva disciplina o un nuevo enfoque para todas las disciplinas? Revista Iberoamericana de Educación, 69 (1), 39-60.

Vilches, A. \& Gil Pérez, D. (2016). La transición a la sostenibilidad como objetivo urgente para la superación de la crisis sistémica actual. Revista Eureka sobre Enseñanza y Divulgación de las Ciencias.13 (2)- pp.395-407. 